Pacific Journal of Mathematics

THE MULTIPLICITY FUNCTIONS OF INVARIANT SUBSPACES
FOR NONSELFADJOINT CROSSED PRODUCTS 


\title{
THE MULTIPLICITY FUNCTIONS OF INVARIANT SUBSPACES FOR NON SELFADJOINT CROSSED PRODUCTS
}

\author{
BARUCH SOLEL
}

Let $\mathcal{L}$ be the von Neumann algebra crossed product determined by a maximal abelian selfadjoint algebra $L^{\infty}(X)$ and an ergodic automorphism of $L^{\infty}(X)$. The algebra $L$ is generated by a bilateral shift $L$ and an abelian algebra $\Re_{L}$ isomorphic to $L^{\infty}(X)$. The non selfadjoint subalge-

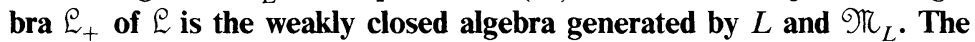
invariant subspaces of $L_{+}$are studied. The notion of multiplicity function is analysed and it is shown that every function $m$ with nonnegative integral values and whose integral, over $X$, is not greater than the measure of $X$, is a multiplicity function. The condition is also a necessary one. We also discuss the notion of canonical models in this setting.

1. Introduction. The invariant subspaces of non selfadjoint crossed products were studied by M. McAsey, P. Muhly and S. K. Saito in [4]. They let $\varrho$ be the von Neumann crossed product determined by a finite von Neumann algebra $M$ and a trace preserving automorphism, and

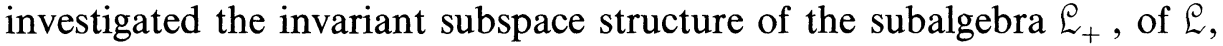
consisting of those operators whose spectrum, with respect to the dual automorphism group on $\mathcal{L}$, is non-negative. The subalgebra $e_{+}$stands,

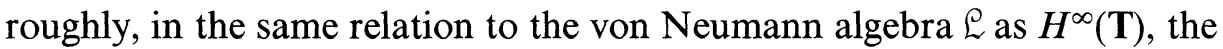
space of boundary values of bounded analytic functions on the unit disc stands in relation to $L^{\infty}(\mathbf{T})$.

Among other results, it is shown, in [4], that $M$ is a factor if and only if a version of the Beurling, Lax. Halmos theorem (analysing the invariant subspaces of the bilateral shift $)$ is valid for $\mathcal{L}_{+}([4$, Theorem 4.1]).

In [3], M. McAsey continued this investigation for the case where $M$ is a maximal abelian selfadjoint algebra. He found conditions for two full, pure invariant subspaces for $\mathcal{L}_{+}$to be unitarily equivalent by a unitary operator in $\mathcal{R}$, the commutant of $\varrho([3$, Theorem 3.5]). To accomplish this, the concept of a multiplicity function was introduced and studied.

In this paper we will study further properties of multiplicity functions in the case where $M$ is $L^{\infty}(X, \mu), \mu$ is a non atomic finite measure and the automorphism is ergodic. We show, in Theorems 3.6 and 3.7, what functions can appear as a multiplicity function of some invariant subspace. 
We will also show (Theorem 4.1) what are the multiplicity functions of the two-sided invariant subspaces (to be defined later) and we shall see that the situation here is somewhat different than in the case when $X$ is a finite set (see [2]). We conclude with remarks about canonical models for the set of the pure invariant subspaces.

2. Definitions and preliminaries. Let $X$ be a standard Borel space with a finite, non atomic measure $\mu$. Let $\tau$ be an invertible measure-preserving ergodic transformation on $X$. Using the product of the counting measure on the integers, $\mathbf{Z}$, and the measure $\mu$ and $X$, we can realize $\mathbf{Z} \times X$ as a measure space. The space $L^{2}(\mathbf{Z} \times X)$, of all (equivalence classes of) functions on $\mathbf{Z} \times X$ satisfying

$$
\sum_{n=-\infty}^{\infty} \int_{X}|f(n, x)|^{2} d \mu(x)<\infty,
$$

is a Hilbert space with inner product

$$
\langle f, g\rangle=\sum_{n=-\infty}^{\infty} \int_{X} f(n, x) \overline{g(n, x)} d \mu(x) \quad\left(f, g \in L^{2}(\mathbf{Z} \times X)\right) .
$$

We shall write $\mathscr{K}$ for this Hilbert space.

Define the following linear, bounded, operators on $\mathscr{K}$ :

$$
\begin{aligned}
& (L f)(n, x)=f\left(n-1, \tau^{-1} x\right), \\
& (R f)(n, x)=f(n-, x), \\
& (L \varphi f)(n, x)=\varphi(x) f(n, x), \quad \varphi \in L^{\infty}(X), \\
& \left(R_{\varphi} f\right)(n, x)=\varphi\left(\tau^{-n} x\right) f(n, x), \quad \varphi \in L^{\infty}(X) .
\end{aligned}
$$

Note that $L$ and $R$ are unitary operators.

Let $\mathfrak{K}_{L}$ (respectively $\mathfrak{K}_{R}$ ) denote the algebra generated by $\left\{L_{\varphi}\right.$ : $\left.\varphi \in L^{\infty}(X)\right\}$ (resp. $\left\{R_{\varphi}: \varphi \in L^{\infty}(X)\right\}$ ). Clearly $\Re_{L}$ and $\Re_{R}$ are abelian von Neumann algebras. The left (resp. right) von Neumann algebra crossed product of $L^{\infty}(X)$ with $\tau$ is defined to be the von Neumann algebra $\mathcal{L}$ (resp. $\Re$ ) generated by $\Re_{L}$ and $L$ (resp. $\Re_{R}$ and $R$ ). Define the left (resp. right) non selfadjoint crossed product to be the weakly closed algebra $\mathcal{L}_{+}$ (resp. $\Re_{+}$) generated by $\Re_{L}$ and $L$ (resp. $\Re_{R}$ and $R$ ).

In this paper we will be interested in subspaces of $\mathscr{K}$ that are invariant under the algebra $\mathfrak{L}_{+}$. Corresponding results hold for $\Re_{+}$-invariant subspaces.

It is known that $\mathcal{L}$ and $\mathcal{R}$ are finite factors satisfying $\mathcal{L}^{\prime}=\mathscr{R}$ and $\Re^{\prime}=\mathcal{L}(\operatorname{see}[\mathbf{1}])$. 
It can be easily seen that, for each integer $n$ and each $\varphi \in L^{\infty}(X)$, $L^{n} L_{\varphi}=L_{\varphi \circ \tau^{-n}} L^{n}$. Hence $L^{n} \Re_{L} L^{-n}=\Re_{L}$ for each $n$ in $\mathbf{Z}$, and $\mathcal{L}$ (resp. $\mathcal{L}_{+}$) is the closure, in the weak operator topology, of the set of operators of the form $\sum_{n=-k}^{k} L_{\varphi_{n}} L^{n}$ (resp. $\sum_{n=0}^{k} L_{\varphi_{n}} L^{n}$ ).

Definitions. A (closed) subspace $\mathscr{K}$ of $\mathscr{K}$ is invariant (or $\mathcal{L}_{+}-$ invariant) if $\mathcal{L}_{+} \Re \subseteq \Re$. It will be $\varrho_{+}$-reducing if $\varrho \Re \subseteq \Re$; pure if $\Re$ is invariant and contains no (non zero) $\mathcal{L}_{+}$-reducing subspace; and full if the smallest $\mathscr{L}_{+}$-reducing subspace containing $\Re$ is $\mathscr{K}$.

The following proposition can be found in [3] or [4].

Proposition 2.1. Let $\Re$ be an invariant subspace in $\mathcal{K}$. Then

(1) $\Re$ reduces $\Re_{L}$;

(2) $\Re$ reduces $\mathcal{L}$ if and only if $\Re$ reduces $L$;

(3) $\Re$ is pure if and only if $\bigcap_{n \geq 0} L^{n} \Re=\{0\}$; and

(4) $\Re$ is full if and only if $\bigvee_{n \leq 0} L^{n} \Re=\mathscr{K}$.

For each closed subspace $\Re \subseteq \mathscr{K}$ we write $P_{\Re}$ for the orthogonal projection whose range is $\Re$. If $\Re$ is an invariant subspace, then $\Re$ reduces $\Re_{L}$, hence $P_{\Re}$ lies in $\Re_{L}^{\prime}$ (the commutant of $\Re_{L}$ ). The subspace $L \Re$ is also invariant (since $\Re_{L} L=L \Re_{L}$ ) and, if we let $\Re$ be $\Re \ominus L \Re$, then $P_{\Re}$ lies in $\Re_{L}^{\prime}$.

Every invariant subspace is an orthogonal sum of an $\mathcal{L}_{+}$-reducing subspace and a pure subspace, and each $\varrho_{+}$-reducing subspace is the range of a projection in $\mathcal{R}$ (see [3, Proposition 3.3 and the remark preceding it]). Therefore, we will be concerned mainly with pure invariant subspaces.

An important tool for dealing with invariant subspaces is the multiplicity function introduced in [2]. To define it, note that the space $\mathscr{K}\left(=L^{2}(\mathbf{Z} \times X)\right)$ may be identified with the direct integral $\int_{X}^{\oplus} l^{2}(\mathbf{Z}) d \mu(x)$, and the algebra $\Re_{L}^{\prime}$, acting on it, may be identified with $\int_{X}^{\oplus} B\left(l^{2}(\mathbf{Z})\right) d \mu(x)$ where $B\left(l^{2}(\mathbf{Z})\right)$ is the algebra of all linear and bounded operators on $l^{2}(\mathbf{Z})$. (For details, see [3]).

Let $\Re$ be an invariant subspace, then $P$, the orthogonal projection on $\Re \ominus L \Re$, lies in $\Re_{L}^{\prime}$ and may be written, using the identification above, as a direct integral $\int_{X}^{\oplus} P(x) d \mu(x)$, where $P(x)$ is a projection in $B\left(l^{2}(Z)\right)$ for almost every $x$ in $X$. We define the multiplicity function, $m$, by letting $m(x)$ be the dimension of the range of $P(x)$. 
The following result is Theorem 3.4 of [3].

THEOREM 2.2. For $i=1,2$ let $\Re_{l}$ be a pure invariant subspace. Let $\Re_{i}$ be $\Re_{i} \ominus L \Re_{i}$ and $m_{i}$ be the multiplicity function of $\Re_{i}$. The following statements are equivalent:

(1) $P_{\Re_{1}}=T P_{\Re_{2}} T^{*}$ for a partial isometry $T \in \Re$, so that $\Re_{1}=T \Re_{2}$.

(2) $m_{1}(x) \leq m_{2}(x)$ a.e.

(3) $P_{\Re_{1}} \preccurlyeq P_{\mathscr{T}_{2}}$ in $\Re_{L}^{\prime}$ (i.e. there is a partial isometry $W$, in $\Re_{L}^{\prime}$, such that $\left.W W^{*}=P_{\vartheta_{1}}, W^{*} W \leq P_{\Re_{2}}\right)$.

Using the arguments that appear in the proof of the theorem above (in [3]) we can also prove the following proposition.

Proposition 2.3. With the notations and assumptions of the preceding theorem, the following statements are equivalent:

(1) $\Re_{1}=T \Re_{2}$ for a partial isometry $T \in \Re$, with initial space that contains $\Re_{2}$.

(2) $m_{1}(x)=m_{2}(x)$ a.e.

(3) $P_{\Re_{1}} \sim P_{\Re_{2}}$ in $\Re_{L}^{\prime}$.

Proof. (1) implies (2): since the initial space of $T$ contains $\Re_{2}, T$ maps $\Re_{2}$ isometrically onto $\Re_{1}$ and $T^{*}$ maps $\Re_{1}$ isometrically onto $\Re_{2}$. Hence $P_{\Re_{1}}=T P_{\Re_{2}}=T^{*} P_{\Re_{1}} T$ and $T^{*} \Re_{1}=\Re_{2}$. Applying Theorem 2.2 we see that $m_{1}(x) \leq m_{2}(x)$ a.e. and also $m_{2}(x) \leq m_{1}(x)$ a.e. (since $\mathfrak{N}_{2}=T \rightarrow \mathfrak{N}_{1}$ and $P_{\mathfrak{N}_{2}}=T^{*} P_{\mathfrak{N}_{1}} T$ ).

Thus $m_{1}(x)=m_{2}(x)$ a.e..

(2) implies (3): Obvious from Theorem 2.2.

(2) implies (1): Suppose $P_{\Re_{1}}=W W^{*}$ and $P_{\Re_{2}}=W^{*} W\left(W \in \Re_{L}^{\prime}\right)$. Then, by Theorem 2.2 , there is a partial isometry $T$, in $\mathcal{R}$, such that $P_{\Re_{1}}=T P_{\pi_{2}} T^{*}$. It follows from the proof of Theorem 2.2 (see [3; Theorem 3.4]) that the initial space of $T$ is $\Sigma_{n=-\infty}^{\infty} \oplus L^{n}\left(W^{*} W^{\Upsilon_{1}}\right)$ and the final space is $\sum_{n=-\infty}^{\infty} \oplus L^{n} \mathcal{K}_{1}$. Since here $W^{*} W=P_{\mathscr{\Re}_{2}}$, the initial space of $T$ is $\sum_{n=-\infty}^{\infty} \oplus L^{n} \mathfrak{K}_{2}$. But $\mathfrak{K}_{2}$ is pure (hence $\bigcap_{n \geq 0} L^{n} \mathfrak{K}_{2}=\{0\}$ ) therefore

$$
\Re_{2}=\underset{n \geq 0}{\vee}\left(\Re_{2} \ominus L^{n} \Re_{2}\right)=\underset{n \geq 0}{\vee}\left(\sum_{k=0}^{n-1} \oplus L^{k} \Re_{2}\right)=\sum_{k=0}^{\infty} \oplus L^{k} \Re_{2}
$$

and $\Re_{2}$ is contained in the initial space of $T$.

REMARK. From the proof of the last implication in the theorem, one can see that if $\Re_{1}\left(\right.$ or $\Re_{2}$ ) is full, then $T T^{*}=I$ (or, respectively, 
$\left.T^{*} T=I\right)$. Since the algebra $R$ is finite, we conclude, in this case, that $T$ is a unitary operator and both $\Re_{1}$ and $\Re_{2}$ are full.

3. Invariant subspaces and the multiplicity function. In this section we will show what are the functions that are multiplicity functions of some pure invariant subspace. This is shown in Theorems 3.6 and 3.7. Before we proceed to prove them we need some lemmas, the most important of which is Lemma 3.5, which enables us to construct an invariant subspace, with a specific multiplicity function, contained in a specific invariant subspace.

LEMMA 3.1. Let $\left\{\mathfrak{T}_{i}\right\}$ be a finite or countable collection of pure invariant subspaces such that $\Re_{1}$ is orthogonal to $\Re_{j}$, whenever $i \neq j$. Let $m_{i}(x)$ be the multiplicity function of $\mathfrak{K}_{i}$. Then the space $\mathfrak{K}=\Sigma \oplus \mathfrak{K}_{i}$ is a pure invariant subspace with multiplicity function $m(x)=\sum m_{i}(x)$ a.e.

Proof. $\Re$ is clearly invariant. To prove that $\Re$ is pure we show that $\wedge_{n \geq 0} L^{n} \Re=\{0\}$. For this, let $x$ be in $\Re$, then $x=\Sigma \oplus x_{i}, x_{i} \in \Re_{i}$. If $x \neq 0$, then one of the $x_{i}-s$, say $x_{1}$, is different from zero, hence there is some $n>0$ such that $x_{1} \notin L^{n} \Re_{1}$, thus $x \notin L^{n} \mathfrak{N}$. Therefore $\Re$ is pure. For the multiplicity function, let $\Re$ be the subspace $\Re \ominus L \Re$ and $\Re_{i}$ be the subspace $\Re_{i} \ominus L \Re_{i}$, so that $\Re=\Sigma \oplus \Re_{i}$ and $P_{\Re}=\Sigma \oplus P_{\Re_{i}}$. For almost every $x$, in $X,\left\{P_{\mathscr{\vartheta}_{i}}(x)\right\}$ is an orthogonal set of projections in $B\left(l^{2}(Z)\right)$ and $P_{\Re(}(x)=\Sigma \oplus^{\prime} P_{\vartheta_{i}}(x)$. Therefore $m(x)=\Sigma m_{i}(x)$.

We say that an invariant subspace $\Re$ is generated by some function $f \in \mathscr{K}$ if $\mathscr{N}$ is the closed linear span of $\left\{L^{n} L_{\varphi} f: \varphi \in L^{\infty}(X), n \geq 0\right\}$; i.e. $\Re$ is the smallest (closed) invariant subspace containing $f$.

LEMMA 3.2. Let $e_{0} \in \mathcal{K}$ be defined as follows: $e_{0}(n, x)=0$ if $n \neq 0$ and $e_{0}(0, x)=\chi_{E}(x)$ (the characteristic function of a measurable set $E$ in $X$ ). Then the invariant subspace $\mathfrak{N}$, generated by $e_{0}$, is pure and its multiplicity function is $\chi_{E}$. We will denote this subspace by $\mathfrak{T}(E)$.

Proof. For a subset $B$ of $\mathbf{Z} \times X$, let $L^{2}(B)$ be the subspace of all the functions in $L^{2}(\mathbf{Z} \times X)$ whose support is contained in $B$. Define $B_{0}=$ $\left\{(k, x) \in \mathbf{Z} \times X ; k \geq 0, x \in \tau^{k}(E)\right\}$. Clearly $e$ lies in $L^{2}\left(B_{0}\right)$. For $g \in \mathscr{K}$, $n>0$, and $\varphi \in L^{\infty}(X)$,

$$
\left(L_{\varphi} L^{n} g\right)(k, x)=\varphi(x) g\left(k-n, \tau^{-n}(x)\right) .
$$


Therefore if $g$ lies in $L^{2}\left(B_{0}\right)$, so does $L \varphi L^{n} g$, and the subspace $L^{2}\left(B_{0}\right)$ is an invariant subspace containing $e_{0}$. Thus $\Re(E) \subseteq L^{2}\left(B_{0}\right)$. Let $g$ be in $L^{2}\left(B_{0}\right) \cap L^{\infty}(\mathbf{Z} \times X)$ and define functions $g_{n} \in L^{\infty}(X)$, for $n>0$, by $g_{n}(x)=g(n, x)$. Then the functions $\left\{L g_{n} L^{n} e_{0}\right\}_{n \geq 0}$ are pairwise orthogonal and their sum is $g$. Thus $g$ lies in $\Re(E)$, and by the density of $L^{2}\left(B_{0}\right) \cap L^{\infty}(\mathbf{Z} \times X)$ in $L^{2}\left(B_{0}\right), L^{2}\left(B_{0}\right)=\Re(E)$.

Now Let $B_{1}$ be the subset $\left\{(k, x) \in B_{0} ; k \geq 1\right\}$, then $L\left(L^{2}\left(B_{0}\right)\right)=$ $L^{2}\left(B_{1}\right)($ by $(*))$ and

$$
\Re \ominus L \Re=L^{2}\left(B_{0}\right) \ominus L^{2}\left(B_{1}\right)=L^{2}\left(B_{0} \backslash B_{1}\right) .
$$

But $B_{0} \backslash B_{1}=\{0\} \times E$, thus the projection onto $\Re \ominus L \Re$ is $\int_{X}^{\oplus} P(x) d \mu(x)$ where $P(x)=0$ if $x \notin E$ and, for $x \in E, P(x)$ is the projection onto the subspace $\mathbf{C} \delta_{0}$, in $l^{2}(\mathbf{Z})$, where $\delta_{0}(n)=0$ if $n \neq 0$ and $\delta_{0}(0)=1$. Thus $m(x)=\operatorname{rank} P(x)=\chi_{E}(x)$.

As noted above, $L\left(L^{2}\left(B_{0}\right)\right)=L^{2}\left(B_{1}\right)$ and, similarly, if we let $B_{n}$ be the set $\left\{(k, x) \in B_{0}: k \geq n\right\}$, then

$$
L^{n}\left(L^{2}\left(B_{0}\right)\right)=L^{2}\left(B_{n}\right)
$$

and

$$
\bigwedge_{n \geq 0} L^{n}(M)=\bigwedge_{n \geq 0} L^{2}\left(B_{n}\right)=L^{2}\left(\bigcap_{n \geq 0} B_{n}\right)=\{0\}
$$

Thus $\Re$ is pure.

Lemma 3.3. Let $E, F$ be measurable subsets of $X$ with $\mu(E)=\mu(F)$. Then there are measurable subsets $\left\{E_{n}\right\}_{n=0}^{\infty}$ and $\left\{F_{n}\right\}_{n=0}^{\infty}$ satisfying:

(1) $E_{n} \subseteq E$ and $F_{n} \subseteq F$ and each $n \geq 0$;

(2) $E_{n} \cap E_{m}=\varnothing$ and $F_{n} \cap F_{m}=\varnothing$ for each $n \neq m$;

(3) $\mu\left(E \backslash \cup_{n=0}^{\infty} E_{n}\right)=0=\mu\left(F \backslash \cup_{n=0}^{\infty} F_{n}\right)$; and

(4) $F_{n}=\tau^{n}\left(E_{n}\right)$ for each $n \geq 0$.

Proof. Define the sets $\left\{E_{n}\right\}_{n=0}^{\infty}$ and $\left\{F_{n}\right\}_{n=0}^{\infty}$ inductively. For $n=0$ let $E_{0}=F_{0}=E \cap F$. For $n=1$ let $F_{1}=\tau\left(E \backslash E_{0}\right) \cap\left(F \backslash F_{0}\right)$ and $E_{1}=$ $\tau^{-1}\left(F_{1}\right)$. When $\left\{E_{n}\right\}_{n=0}^{k}$ and $\left\{F_{n}\right\}_{n=0}^{k}$ are defined we let $F_{k+1}$ be $\tau^{k}\left(E \backslash \cup_{n=0}^{k} E_{n}\right) \cap\left(F \backslash \cup_{n=0}^{k} F_{n}\right)$ and $E_{k+1}=\tau^{-k-1}\left(F_{k+1}\right)$. The sets we get in this manner clearly satisfy (1), (2) and (4). For each $k>0$,

$$
F_{k+1}=\tau^{k}\left(E \backslash \bigcup_{n=0}^{k} E_{n}\right) \cap\left(F \backslash \bigcup_{n=0}^{k} F_{n}\right)
$$


and

$$
\begin{aligned}
& \varnothing=\tau^{k}\left(E \backslash \bigcup_{n=0}^{k} E_{n}\right) \cap\left(F \backslash \bigcup_{n=0}^{k+1} F_{n}\right) \\
& \supseteq \tau^{k}\left(E \backslash \bigcup_{n=0}^{\infty} E_{n}\right) \cap\left(F \backslash \bigcup_{n=0}^{\infty} F_{n}\right) .
\end{aligned}
$$

Hence, let $E^{\prime}$ be $E \backslash \cup_{n=0}^{\infty} E_{n}$ and $F^{\prime}$ be $F \backslash \cup_{n=0}^{\infty} F_{n}$, then $\tau^{k}\left(E^{\prime}\right) \cap F^{\prime}$ $=\varnothing$ for each $k>0$. It follows, from the ergodicity of $\tau$, that either $\mu\left(E^{\prime}\right)=0$ or $\mu\left(F^{\prime}\right)=0$. But

$$
\mu\left(E^{\prime}\right)=\mu(E)-\sum_{n=0}^{\infty} \mu\left(E_{n}\right)=\mu(F)-\sum_{n=0}^{\infty} \mu\left(F_{n}\right)=\mu\left(F^{\prime}\right) .
$$

Hence $\mu\left(E^{\prime}\right)=\mu\left(F^{\prime}\right)=0$.

LEMMA 3.4. If $E, F$ and $\left\{E_{n}\right\}_{n=0}^{\infty},\left\{F_{n}\right\}_{n=0}^{\infty}$ are as in the preceding lemma, then:

(1) The operator $U=\sum_{k=0}^{\infty} L_{\chi_{F_{k}}} L^{k}$ is a partial isometry in $\mathcal{L}_{+}$with initial projection $L_{\chi_{E}}$ and final projection $L_{\chi_{F}}$; and

(2) the operator $V=\sum_{k=0}^{\infty} R_{\chi_{E_{k}}} R^{k}$ is a partial isometry in $\Omega_{+}$with initial projection $R_{\chi_{F}}$ and final projection $R_{\chi_{E}}$.

Proof. It can be easily checked that, for each $n \in Z$ and $k \geq 0$,

$$
L^{n} L_{\chi_{F_{k}}} L^{-n}=L_{\chi_{\tau^{-n}\left(E_{k}\right)}} \text { and } \quad R^{n} R_{\chi_{E_{k}}} R^{-n}=R_{\chi_{\tau^{-n}\left(E_{k}\right)}} .
$$

Therefore $L_{\chi_{F_{k}}} L^{k}$ is a partial isometry with initial projection $L_{\chi_{\tau}-k\left(F_{k}\right)}$ and final projection $L_{\chi_{F_{k}}}$. Since $L_{\chi_{\tau}-k\left(F_{k}\right)}=L_{\chi_{F_{k}}}$ and $\left\{L_{\chi_{F_{k}}}\right\},\left\{L_{\chi_{F_{k}}}\right\}$ are orthogonal families of projections, $U$ is a partial isometry with initial projection $\sum_{k=0}^{\infty} L_{\chi_{E_{k}}}=L_{\chi_{E}}$ and final projection $\sum_{k=0}^{\infty} L_{\chi_{F_{k}}}=L_{\chi_{F}}$. It is in $\mathcal{L}_{+}$because the sum is in the sense of convergence in the strong operator topology and each of the operators $L_{\chi_{F_{k}}} L^{k}$ is in $\mathcal{L}_{+}$.

The statement for $V$ is proved similarly.

LEMMA 3.5. Let $\Re$ be a pure invariant subspace with multiplicity function $m(x)=\chi_{E}(x)$, for some measurable subset $E$, in $X$. Let $F \subseteq X$ be a measurable set with $\mu(F)=\mu(E)$. Then there is a pure invariant subspace $\Re$, contained in $\Re$, with multiplicity function $\chi_{F}$.

Proof. Let $\Re(E)$ be the pure invariant subspace given by Lemma 3.2. Since $m(x)=\chi_{E}(x)$, Proposition 2.3 applies to give a partial isometry $T$, in $\Re$, whose initial space contains $\mathscr{R}(E)$, its final space contains $\Re$, and 
$\Re=T \Re(E)$. Since $\Re(E)$ is the closed linear span of the set $\left\{L^{n} L_{\varphi} e_{0}\right.$ : $\left.\varphi \in L^{\infty}(X), n \geq 0\right\}$, and $T$ commutes with $\mathcal{L}$, $\Re$ is generated by $T e_{0}$ (recall that $e_{0}(n, x)=0$ if $n \neq 0$ and $e_{0}(0, x)=\chi_{E}(x)$ ).

We assumed that $\mu(E)=\mu(F)$, hence we can use Lemma 3.4 to define the partial isometries

$$
U=\sum_{k=0}^{\infty} L_{\chi_{F_{k}}} L^{k} \text { and } \quad V=\sum_{k=0}^{\infty} R_{\chi_{E_{k}}} R^{k}
$$

where $\left\{E_{k}\right\}_{k=0}^{\infty}$ and $\left\{F_{k}\right\}_{k=0}^{\infty}$ are given by Lemma 3.3.

Let $f_{0} \in \mathcal{K}$ be defined by: $f_{0}(n, x)=0$ if $n \neq 0$ and $f_{0}(0, x)=\chi_{F}$. Then, using properties of $\left\{E_{n}\right\}_{n=0}^{\infty}$ and $\left\{F_{n}\right\}_{n=0}^{\infty}$,

$$
\begin{aligned}
\left(U e_{0}\right)(k, x) & =\sum_{n=0}^{\infty} \chi_{F_{n}}(x)\left(L^{n} e_{0}\right)(k, x)=\chi_{F_{k}}(x) \chi_{E}\left(\tau^{-k}(x)\right) \\
& =\chi_{F_{k}}(x) \chi_{\tau^{k}(E)}(x)=\chi_{F_{k}}(x)
\end{aligned}
$$

and

$$
\left(V f_{0}\right)(k, x)=\sum_{k=0}^{\infty} \chi_{E_{n}}\left(\tau^{-k}(x)\right)\left(R^{n} f_{0}(k, n)\right)=\chi_{\tau^{k}\left(E_{k}\right)}(x) \chi_{F}(x)=\chi_{F_{k}}(x) .
$$

If we now define $f(n, x)=\chi_{F_{n}}(x)$, we have that $f=U e_{0}=V f_{0}$.

Let $\Re_{1}$ be the invariant subspace generated by $f$. The initial projection of $V$ is $r_{\chi_{F}}$ and we have

$$
\left(R_{\chi_{F}} f_{0}\right)(n, x)=\chi_{F}\left(\tau^{-n}(x)\right) f_{0}(n, x)=f_{0}(n, x) .
$$

Thus the initial space of $V$ (which is an invariant subspace, since $V \in \Re$ $\left.=\theta^{\prime}\right)$ contains $f_{0}$, and hence contains $\Re(F)$ (the invariant subspace generated by $\left.f_{0}\right)$. Therefore $V$ maps $\Re(F)$ isometrically onto $\Re_{1}$ and, since $V$ lies in $\Re$, it also maps $L^{n} \Re(F)$ isometrically onto $L^{n} \Re_{1}$, for each $n \geq 0$. The subspace $\Re(F)$ is pure (i.e. $\wedge_{n \geq 0} L^{n} \Re(F)=\{0\}$ ), hence so is $\Re_{1}$.

Applying Proposition 2.3, for $\Re(F)$ and $\Re_{1}$, we find that the multiplicity function, $n_{1}(x)$, of $\Re_{1}$ equals $\chi_{F}(x)$ almost everywhere.

Now, let $\Re$ be the subspace $T \Re_{1}$. Since $\mathcal{K}_{1}$ is generated by $f\left(=U e_{0}\right)$ and $U$ lies in $\mathscr{L}_{+}$, we see that $\Re_{1} \subseteq \Re(E)$ and, therefore, $T$ maps $\Re_{1}$ isometrically onto $\mathcal{X}$. It also maps $L^{n} \mathcal{K}_{1}(n \geq 0)$ isometrically onto $L^{n} \mathfrak{X}$

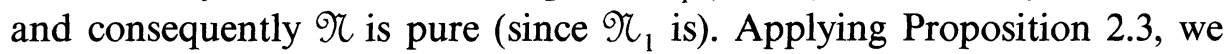
find that the multiplicity function of $\Re$ equals $\chi_{F}$ almost everywhere.

Since $\Re_{1} \subseteq \Re(E), \Re=T \Re \Re_{1} \subseteq T \Re(E)=\mathscr{K}$ and we are done. 
REMARK. With the notations of the lemma, $e_{0}=U^{*} f$ and $f=U e_{0}$. Since $U$ lies in $\Re\left(=\mathcal{L}^{\prime}\right)$,

$$
V\left\{L^{n} \Re(E): n \in \mathbf{Z}\right\}=e e_{0}=e f=V\left\{L^{n} \Re_{1}: n \in \mathbf{Z}\right\} .
$$

The initial space of $T$ is a range of a projection in $\mathcal{R}$ and contains $\mathscr{R}(E)$, hence contains $\mathcal{L} e_{0}$. It follows that

$$
\begin{aligned}
& V\left\{L^{n} \Re: n \in Z\right\}=V\left\{T L^{n} \Re(E): n \in \mathbf{Z}\right\}=T £ e_{0}=T £ f \\
& =V\left\{T L^{n} \Re_{1}: n \in \mathbf{Z}\right\}=V\left\{L^{n} \Re: n \in \mathbf{Z}\right\} \text {. }
\end{aligned}
$$

THEOREM 3.6. If $m(x)$ is a measurable function on $X$ with values in $Z_{+}$ (the non-negative integers) and $\int_{X} m(x) d \mu(x) \leq \mu(X)$, then there is a pure invariant subspace $\mathfrak{T}_{\mathrm{L}}$ with multiplicity function $m(x)$. Moreover, if $\int_{X} m(x) d \mu(x)=\mu(X)$ then we can find such a subspace $\mathfrak{R}$ that is also full.

Proof. We can write $m(x)=\sum_{n=1}^{\infty} \chi_{E_{n}}$, where $\left\{E_{n}\right\}_{n=1}^{\infty}$ are measurable sets (not necessarily pairwise disjoint) and $\sum_{n=1}^{\infty} \mu\left(E_{n}\right) \leq \mu(X)$. Since $\mu$ is non atomic, we can find subsets $\left\{F_{n}\right\}_{n=1}^{\infty}$ of $X$, pairwise disjoint, such that $\mu\left(E_{n}\right)=\mu(F)$ for each $n \geq 1$. Let $B_{n}$ be the set $\{(k, x) \in \mathbf{Z} \times X ; k \geq 0$, $\left.x \in \tau^{k}\left(F_{n}\right)\right\}$. Then it follows from the proof of Lemma 3.2 that $\Re\left(F_{n}\right)=$ $L^{2}\left(B_{n}\right) n \geq 1$. Since the sets $\left\{F_{n}\right\}_{n=1}^{\infty}$ are pairwise disjoint, so are $\left\{B_{n}\right\}_{n=1}^{\infty}$ and the subspaces $\Re\left(F_{n}\right)_{n=1}^{\infty}$ are pairwise orthogonal. By Lemma 3.1, the subspace $\Re=\Sigma \oplus \Re\left(F_{n}\right)$ is a pure invariant subspace with multiplicity function that equals $\sum_{n=1}^{\infty} \chi_{F_{n}}$ almost everywhere.

Applying Lemma 3.5, we find, for each $n \geq 1$, a pure invariant subspace $\Re_{n}$, contained in $\Re\left(F_{n}\right)$, with multiplicity function that equals $\chi_{E_{n}}$ almost everywhere. By Lemma 3.1, the subspace $\mathfrak{K}=\Sigma \oplus \mathfrak{K}_{n}$ is a pure invariant subspace with multiplicity function $m(x)\left(=\Sigma \chi_{E_{n}}\right)$.

For the last assertion, note that, when $\int_{X} m(x) d \mu(x)=\mu(X)$, $\cup_{n=1}^{\infty} F_{n}=X$ and therefore $\cup_{n=1}^{\infty} B_{n}=\mathbf{Z}_{+} \times X$ and $\mathscr{\mathcal { C }}=L^{2}\left(\mathbf{Z}_{+} \times X\right)$.

But, for each $n \geq 1, \bigvee\left\{L^{k} \Re_{n}: k \in \mathbf{Z}\right\}=\bigvee\left\{L^{k} \Re\left(F_{n}\right): k \in \mathbf{Z}\right\}$ (see the remark following Lemma 3.5) and then,

$$
\begin{aligned}
\bigvee\left\{L^{k} \Re: k \in \mathbf{Z}\right\} & =\bigvee\left\{L^{k} \Re_{n}: k \in \mathbf{Z}, n \geq 1\right\} \\
& =\bigvee\left\{L^{k} \Re\left(F_{n}\right): k \in \mathbf{Z}, n \geq 1\right\}=\bigvee\left\{L^{k} \mathfrak{T}: k \in \mathbf{Z}\right\} .
\end{aligned}
$$

Since $\Re=L^{2}\left(\mathbf{Z}_{+} \times X\right), \quad L^{k} \Re=L^{2}(\{(n, x): \quad x \in X, n \geq k\})$ and $\bigvee\left\{L^{k} \Re: k \in \mathbf{Z}\right\}=L^{2}(\mathbf{Z} \times X)$. Hence $\mathscr{N}$, and also $\Re$, is full.

We shall show now that the condition $\int_{X} m(x) d \mu(x) \leq \mu(X)$ is necessary, in order to find a pure invariant subspace with multiplicity function $m(x)$. 
THEOREM 3.7. Let T be a pure invariant subspace with multiplicity function $m(x)$. Then $\int_{X} m(x) d \mu(x) \leq \mu(X)$ and equality holds if and only if Th is full.

Proof. Suppose $\int_{X} m(x) d \mu(x) \geq \mu(X)$. Then, since the measure $\mu$ is non atomic, we can find another measurable function $m_{1}(x)$, with nonnegative integral values, such that $m_{1}(x) \leq m(x)$ and $\int_{X} m_{1}(x) d \mu(x)=$ $\mu(X)$ (and if $\int_{X} m(x) d \mu(x)=\mu(X)$, then $m_{1}(x)=m(x)$ ). Applying the preceding theorem we can find a pure and full invariant subspace $\mathfrak{K}_{1}$ with multiplicity function $m_{1}(x)$.

Since $m_{1}(x) \leq m(x)$ we can use Theorem 2.2 to obtain a partial isometry $T$, in $\Re$, such that $\Re_{1}=T \Re$. The final space of $T$ is the range of a projection in $R$, and, since it contains $\Re_{1}$, it contains $\bigvee\left\{L^{n} \Re_{1}: n \in \mathbf{Z}\right\}$. But $\Re_{1}$ is full, hence the final space of $T$ is $\mathscr{K}$ and, by finiteness of the algebra $\Re, T$ is a unitary operator. Applying Proposition 2.3 we have $m(x)=m_{1}(x)$ a.e. and, therefore, $\int_{X} m(x) d \mu(x)=$ $\mu(X)$.

If $\int_{X} m(x) d \mu(x)=\mu(X)$ then, as we have seen above, $\Re=T^{*} \Re_{1}$ where $\mathfrak{T}_{1}$ is the subspace constructed in Theorem 3.6 (for the function $m(x))$. Therefore $\Re_{1}$ is full and

$$
\begin{aligned}
\vee\left\{L^{n} \Re: n \in \mathbf{Z}\right\} & =\vee\left\{T^{*} L^{n} \Re_{1}: n \in \mathbf{Z}\right\} \\
& =T^{*} \vee\left\{L^{n} \Re_{1}: n \in \mathbf{Z}\right\}=\mathscr{K} .
\end{aligned}
$$

Hence $\Re$ is full.

On the other hand, suppose $\Re$ is full. Since $\int_{X} m(x) d \mu(x) \leq \mu(X)$, we can find a measurable function $m_{1}(x)$, with values in $\mathbf{Z}_{+}$, such that $m_{1}(x) \geq m(x)$ and $\int_{X} m_{1}(x) d \mu(x)=\mu(X)$. Using Theorem 3.6 and Theorem 2.2 we can find a pure and full invariant subspace $\Re_{1}$, with multiplicity function $m_{1}(x)$, and a partial isometry $T$, in $\Re$, such that $\Re=T \Re_{1}$. The final space of $T$ contains $\Re$, a full invariant subspace, hence $T$ is a unitary operator and, by Proposition 2.3, $m(x)=m_{1}(x)$ a.e. and $\int_{X} m(x) d \mu(x)=\mu(X)$.

4. Two-sided invariant subspaces and canonical models. Define a subspace $\Re$ of $\mathscr{K}$ to be two-sided invariant if it is an invariant subspace for the algebra $\mathscr{L}_{+} \vee \Re_{+}$, the weak-operator closed algebra generated by $\varrho_{+}$and $\Re_{+}$.

In [2] M. McAsey introduced the notion of canonical models for invariant subspaces. A complete set canonical models was defined to be a 
family of full, pure invariant subspaces $\left\{\mathscr{T l}_{i}\right\}_{i \in I}$ with:

(a) for no two distinct indices $i$ and $j$ is $P_{\Re_{1}}$ unitarily equivalent to $P_{\Re_{1}}$ by a unitary operator in $R$; and

(b) for every pure invariant subspace $\Re$, there is an $i$ in $I$ and a partial isometry $T$ in $\Re$ such that $T P_{\Re_{l}} T^{*}=P_{\Re}$ (in particular $\Re=T \Re_{l}$ ).

In the setting of [2], the space $X$ is a finite set, $X=\left\{x_{i}: 0 \leq i \leq n-1\right\}$ (with the counting measure $\mu$ ) and $\tau\left(x_{i}\right)=x_{i+1}(i \neq n-1), \tau\left(x_{n-1}\right)=x_{0}$. The definitions of the algebras $\mathcal{L}$ and $\mathcal{R}$ and the notions of invariance and multiplicity function are analogous to the ones in our setting.

It is shown there ([2, Theorem 4.1]) that a finite set of two-sided invariant subspaces can be used as a set of canonical models for the pure invariant subspaces. In order to establish this result one has to show that for each measurable function $m$ on $X$ having values in $Z_{+}$and satisfying $\int_{X} m(x) d \mu(x)=\mu(X)$ (when $X$ is a finite set $\sum_{i=1}^{n} m\left(x_{i}\right)=n$ ) there is a two-sided invariant subspace with multiplicity function $m$. This is proved in Theorem 3.4 of [2].

In the setting of our study this cannot always be done and, as we shall soon see, the existence of such a subspace, for $m(x)$, is related to whether $1-m(x)$ is a coboundary or not.

THEOREM 4.1. Let $m(x)$ be a measurable function with values in $\mathbf{Z}_{+}$and satisfying $\int_{X} m(x) d \mu(x)=\mu(X)$. Then there is a two-sided invariant subspace $\Re$, with multiplicity function $m(x)$, if and only if there is a measurable function $d$, on $X$, with values in $\mathbf{Z}$, such that

$$
d(x)-d\left(\tau^{-1}(x)\right)=1-m(x) \quad \text { a.e. }
$$

Proof. Suppose, first, that such a subspace $\Re$ exists. It is shown in [3, Theorem 4.3] that there is a subset $B$, in $\mathbf{Z} \times X$, satisfying $\lambda(B) \subseteq B$ and $\rho(B) \subseteq B$ where $\lambda(k, x)=(k+1, \tau(x)), \rho(k, x)=(k+1, x)$, and $\Re$ $=L^{2}(B) \quad(=\{f \in \mathcal{K}: f(k, x)=0$ for $(k, x) \notin B\})$. Define $d(x)=$ $\inf \{k:(k, x) \in B\}$ where inf $\varnothing=\infty$ and $\inf \mathbf{Z}=-\infty$.

Let $C_{1}$ be the set $\{x \in X:(k, x) \in B$ for each $k \in \mathbf{Z}\}$ and $C_{2}$ be the set $\{x \in X:(k, x) \in B$ for some $k \in \mathbf{Z}\}$. Since $\lambda(B) \subseteq B$, we have that if $(k, x)$ lies in $B$ then $(k+1, \tau(x))$ is in $B$. Therefore $\tau\left(C_{l}\right) \subseteq C_{i}, i=1,2$. It follows from the ergodicity of $\tau$ that, for each $i=1,2$, either $\mu\left(C_{i}\right)=0$ or $\mu\left(X \backslash C_{l}\right)=0$. If $\mu\left(X \backslash C_{1}\right)=0$ then $(k, x)$ lies in $B$, for every $k \in Z$ and almost every $x \in X$. Hence $\mathscr{K}=L^{2}(B)=\mathscr{K}$ in this case and the multiplicity function would be zero almost everywhere. As $\int_{X} m(x) d \mu(x)$ $=\mu(X)$, this cannot occur and, so, $\mu\left(C_{1}\right)=0$ and $d(x)>-\infty$ a.e. 
If $\mu\left(C_{2}\right)=0$, then $\Re=L^{2}(B)=\{0\}$. This also contradicts the assumption about $m$ and we have that $\mu\left(X \backslash C_{2}\right)=0$ and $d(x)<\infty$ a.e.

It follows that we can, by changing $d$ on a set of measure zero, assume that $d$ is finite everywhere. To see that $(*)$ holds we let $A$ be the set $B \backslash \lambda(B)$ and $A_{x}$, for $x \in X$, be the set $\{k \in \mathbf{Z}:(k, x) \in A\}$. Since ף $=L^{2}(B)$, we have $L \Re=L L^{2}(B)=L^{2}(\lambda(B))$ and

$$
\mathfrak{M} \ominus L \Re=L^{2}(B \backslash \lambda(B))=L^{2}(A)=\int_{X}^{\oplus} l_{2}\left(A_{x}\right) d \mu(x) \text {. }
$$

Therefore $m(x)=$ number of elements in $A_{x}$ (to be denoted by $\#\left(A_{x}\right)$ ).

Note that, from the definition of $d(x)$, if $(k, x)$ lies in $B$ then $d(x) \leq k$. Also, if $d(x) \leq k$, then $(d(x), x)$ lies in $B$ and

$$
(k, x)=\rho^{k-d(x)}(d(x), x) \in \rho^{k-d(x)}(B) \subseteq B .
$$

Hence $B=\{(k, x): d(x) \leq k\}$ and $\lambda(B)=\left\{(k, x): d\left(\tau^{-1}(x)\right) \leq k-1\right\}$. Thus

$$
\begin{aligned}
A & =\left\{(k, x): d(x) \leq k<1+d\left(\tau^{-1}(x)\right)\right\}, \\
A_{x} & =\left\{k \in \mathbf{Z}: d(x) \leq k<1+d\left(\tau^{-1}(x)\right)\right\}, \\
\#\left(A_{x}\right) & =1+d\left(\tau^{-1}(x)\right)-d(x) .
\end{aligned}
$$

Thus $m(x)=1+d\left(\tau^{-1}(x)\right)-d(x)$.

To prove the converse, suppose that $d$ satisfies $(*)$. Let $B$ be the set $\{(k, x): d(x) \leq k\}$, then

$$
\begin{aligned}
\lambda(B) & =\left\{(k, x): d\left(\tau^{-1}(x)\right) \leq k-1\right\} \\
& =\{(k, x): d(x)+m(x)-1 \leq k-1\},
\end{aligned}
$$

and $\lambda(B)$ is contained in $B$ since $m(x) \geq 0$. We also have $\rho(B) \subseteq B$ because $\rho(B)=\{(k, x): d(x) \leq k-1\} \subseteq B$.

Hence the subspace $\Re=L^{2}(B)$ is a two-sided invariant subspace (see Theorem 4.3 of [3]) and $\Re \ominus \Re=L^{2}(B \backslash \lambda(B))\left(=L^{2}(A)\right)$. Again,

$$
\begin{aligned}
A_{x} & =\left\{k \in \mathbf{Z}: d(x) \leq k<1+d\left(\tau^{-1}(x)\right)\right\} \\
& =\{k \in \mathbf{Z}: d(x) \leq k<d(x)+m(x)\}
\end{aligned}
$$

and the multiplicity function of $\Re$ is $m(x)$.

We cannot, therefore, find a complete set of canonical models among the two-sided invariant subspaces. We can, however, find a complete set of canonical models among the pure and full invariant subspaces. 
For each measurable function $m(x)$, with values in $\mathbf{Z}_{+}$, satisfying $\int_{X} m(x) d \mu(x)=\mu(X)$, we can, using Theorem 3.6, construct a pure, full, invariant subspace $\Re$ with multiplicity function $m(x)$. The set obtained in this manner, to be denoted by $\mathscr{F}$, can serve as a complete set of canonical models. Indeed, for each pure invariant subspace $\mathfrak{N}$, with multiplicity function $m$, there is some measurable function $m_{1}(x)$, with values in $\mathbf{Z}_{+}$and such that $m_{1}(x) \geq m(x)$ almost everywhere and $\int_{X} m_{1}(x) d \mu(x)=\mu(X)$. Using Theorem 2.2 we can find a partial isometry $T \in \Re$ such that $P_{\Re}=T P_{\mathscr{N}_{1}} T^{*}$ where $\mathfrak{N}_{1}$ is the subspace in $\mathscr{F}$ having multiplicity function $m_{1}(x)$ almost everywhere.

We conclude by pointing out that the ergodicity of $\tau$ (assumed throughout the paper) is necessary for Theorem 3.6 to hold.

Proposition 4.2. If $\tau(E)=E$ for a subset $E$, of $X$, with $\mu(E)>0$, $\mu(X \backslash E)>0$, then there is no pure invariant subspace $\mathfrak{T}$ with multiplicity function $m(x)=\chi_{E}(x)+\chi_{F}(x)$ where $F \subseteq E$ is a subset of positive measure (even if $\left.\int_{X} m(x) d \mu(x)<\mu(X)\right)$.

Proof. Let $Q$ be the set $X \backslash E$. Then $L^{2}(\mathbf{Z} \times X)=L^{2}(\mathbf{Z} \times E) \oplus$ $L^{2}(\mathbf{Z} \times Q)$. Denote $L^{2}(\mathbf{Z} \times E)$ by $\mathcal{K}_{1}$ and $L^{2}(\mathbf{Z} \times Q)$ by $\mathscr{K}_{2}$. Then

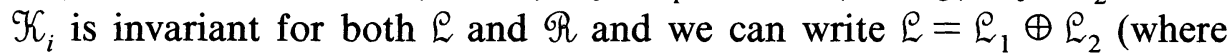
$\left.\mathcal{L}_{i}=\mathcal{L} \mid \mathcal{K}_{i}\right)$ and $\mathscr{R}=\mathscr{R}_{1} \oplus \Re_{2}\left(\right.$ where $\left.\mathscr{R}_{i}=\Re \mid \mathscr{K}_{i}\right)$.

If $\mathscr{N} \subseteq \mathscr{K}$ is a pure invariant subspace, then $\mathscr{N}=\mathfrak{K}_{1} \oplus \mathfrak{K}_{2}$ where $\mathscr{K}_{i}$ is a pure invariant subspace in $\mathscr{K}_{i}$ (invariant with respect to $\left.\mathscr{L}_{+} \mid \mathscr{K}_{i}\right)$ because each $\mathcal{K}_{i}$ is the range of a central projection. If $m(x)$ is the multiplicity function of $\Re$ then, as one can easily see, $m_{1}(x)=\chi_{E}(x) m(x)$ and $m_{2}(x)=\chi_{Q}(x) m(x)$ are the multiplicity of $\Re_{1}$ and $\Re_{2}$.

If we assume that $m(x)=\chi_{E}(x)+\chi_{F}(x), F \subseteq E$, and $\mu(F)>0$ then $m_{1}(x)=\chi_{E}(x)+\chi_{F}(x)$ and, hence, restricting our attention to $\mathscr{K}_{1}$, we see that $\mathfrak{R}_{1}$ is a pure invariant subspace with multiplicity function greater than one. Since the multiplicity function of $L^{2}\left(\mathbf{Z}_{+} \times E\right.$ ) (as a subspace of $\mathcal{K}_{1}$ ) is identically one, Theorem 2.2 implies that there is a partial isometry $T$, in $\Re_{1}$, that maps $\Re_{1}$ onto $L^{2}\left(\mathbf{Z}_{+} \times E\right)$ (note that Theorem 2.2 and Proposition 2.3 do not use ergodicity and, hence, might be used here). The range subspace of $T$ contains $L^{2}\left(\mathbf{Z}_{+} \times E\right)$ and the corresponding projection lies in $\Re$, hence the range of $T$ is $\mathscr{K}_{1}$ and, since $\Re$ is finite, $T$ is a unitary operator. This shows that $m_{1}=1$ (using Proposition 2.3 for $\mathfrak{K}_{1}$ and $L^{2}\left(\mathbf{Z}_{+} \times E\right)$ ) and, thus, contradicts our assumption. 


\section{REFERENCES}

[1] J. Dixmier, Les algèbras d'opérateurs dans l'espace Hilbertien, Gauthier-Villars, Paris 1969.

[2] M. McAsey, Canonical modesl for invariant subspaces. Pacific J. Math., 91 (1980), 377-395.

[3] _ Invariant subspaces of non selfadjoint crossed products, Pacific J. Math., 96 (1981), 457-473.

[4] M. McAsey, P. S. Muhly and K.-S. Saito, Nonselfadjoint crossed products (invariant subspaces and maximality). Trans. Amer. Math. Soc., 248 (1979), 381-409.

[5] F. J. Murray and J. von Neumann, On rings of operators. Ann. of Math., (2) 37 (1936), 116-229.

Received May 25, 1982.

TeL-AVIV UnIVERSITY

TEL-Aviv 69978, ISRAEL 


\section{PACIFIC JOURNAL OF MATHEMATICS \\ EDITORS}

Donald BABBITT (Managing Editor)

University of California

Los Angeles, CA 90024

Hugo Rossi

University of Utah

Salt Lake City, UT 84112

C. C. Moore and Arthur Ogus

University of California

Berkeley, CA 94720
J. DugunduI

Department of Mathematics

University of Southern California

Los Angeles, CA 90089-1113

R. FinN and H. SAMELSON

Stanford University

Stanford, CA 94305

ASSOCIATE EDITORS

R. ARENS

E. F. BECKENBACH

B. H. NeUMANN

F. WOLF

K. YosHIDA (1906-1982)

\section{SUPPORTING INSTITUTIONS}

UNIVERSITY OF ARIZONA

UNIVERSITY OF BRITISH COLUMBIA

CALIFORNIA INSTITUTE OF TECHNOLOGY

UNIVERSITY OF CALIFORNIA

MONTANA STATE UNIVERSITY

UNIVERSITY OF NEVADA, RENO

NEW MEXICO STATE UNIVERSITY

OREGON STATE UNIVERSITY
UNIVERSITY OF OREGON

UNIVERSITY OF SOUTHERN CALIFORNIA

STANFORD UNIVERSITY

UNIVERSITY OF HAWAII

UNIVERSITY OF TOKYO

UNIVERSITY OF UTAH

WASHINGTON STATE UNIVERSITY

UNIVERSITY OF WASHINGTON 


\section{Pacific Journal of Mathematics}

\section{Vol. 113, No. $1 \quad$ March, 1984}

Flavio E. A. da Silveira, Rational homotopy theory of fibrations $\ldots \ldots \ldots \ldots 1$

Donald M. Davis, Desuspensions of stunted projective spaces ............ 35

Lou van den Dries, Exponential rings, exponential polynomials and

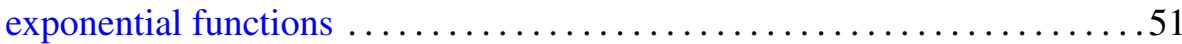

Fred Galvin and Samuel David Shore, Completeness in semimetric

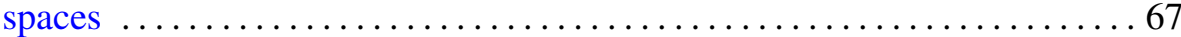

Fereidoun Ghahramani, Compact elements of weighted group algebras . . . 777

Munehiko Itōo, The closed image of a hereditary $M_{1}$-space is $M_{1} \ldots \ldots \ldots 85$

Elvira Laura Livorni, Classification of algebraic surfaces with sectional genus less than or equal to six. I. Rational surfaces .............. 93

H. Alan MacLean, Riesz sets and a theorem of Bochner ............ 115

E. Neher, Jordan triple systems with completely reducible derivation or

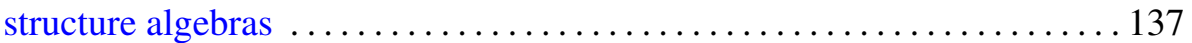

Joe Repka, Shalika's germs for $p$-adic GL( $n)$. I. The leading term $\ldots \ldots \ldots 165$

Joe Repka, Shalika's germs for $p$-adic GL( $n)$. II. The subregular term $\ldots \ldots 173$

Rae Michael Andrew Shortt, Borel density, the marginal problem and isomorphism types of analytic sets $\ldots \ldots \ldots \ldots \ldots \ldots \ldots \ldots \ldots \ldots \ldots \ldots \ldots$

Baruch Solel, The multiplicity functions of invariant subspaces for nonselfadjoint crossed products $\ldots \ldots \ldots \ldots \ldots \ldots \ldots \ldots \ldots \ldots \ldots \ldots . \ldots \ldots$

Su-win Yang, Self-intersection number of immersions and enumeration of nonstable vector bundles

W. M. Zajączkowski, Local solvability of nonstationary leakage problem for ideal incompressible fluid. II 